\title{
Mini-Review
}

\section{Drug Absorption Modeling as a Tool to Define the Strategy in Clinical Formulation Development}

\author{
Martin Kuentz ${ }^{1,2}$
}

Received 30 May 2008; accepted 30 July 2008; published online 27 August 2008

\begin{abstract}
The purpose of this mini review is to discuss the use of physiologically-based drug absorption modeling to guide the formulation development. Following an introduction to drug absorption modeling, this article focuses on the preclinical formulation development. Case studies are presented, where the emphasis is not only the prediction of absolute exposure values, but also their change with altered input values. Sensitivity analysis of technologically relevant parameters, like the drug's particle size, dose and solubility, is presented as the basis to define the clinical formulation strategy. Taking the concept even one step further, the article shows how the entire design space for drug absorption can be constructed. This most accurate prediction level is mainly foreseen once clinical data is available and an example is provided using mefenamic acid as a model drug. Physiologically-based modeling is expected to be more often used by formulators in the future. It has the potential to become an indispensable tool to guide the formulation development of challenging drugs, which will help minimize both risks and costs of formulation development.
\end{abstract}

KEYWORDS: clinical; design space; drug absorption; formulation; parameter sensitivity; physiologicallybased modelling.

\section{INTRODUCTION}

Today's pharmaceutical Industry is facing a productivity crisis. Fewer drugs are approved despite constantly increasing development costs (1). Research-based companies have to decrease their attrition rates to cope with the situation and to retain their competitiveness (2). One of the key problems is to overcome the biopharmaceutical hurdle that is raised by poorly soluble drug candidates. The issue can be addressed by an early ADME profiling to better assess the developability of a drug (3). Such a profiling is nowadays not only conducted by means of in vitro experiments, but also by computational methods $(4,5)$. The well known Lipinski rules (6) can help in anticipating drug absorption hurdles and more recently, some quantitative structure bioavailability relationships (QSBR) were proposed leading to a more refined estimate of the drug absorption (7-15). An alternative to the QSBR approach is the concept of the absorption potential (16) or the estimation of a maximal absorbable dose (17).

A reliable prediction of a drug's absorption and/or bioavailability is not only important for the selection of a candidate, but is equally critical for its subsequent pharmaceutical development. However, in the advanced phase of

\footnotetext{
${ }^{1}$ University of Applied Sciences Northwestern Switzerland, Institute of Pharma Technology, Gründenstr., 4132, Muttenz, Switzerland.

${ }^{2}$ To whom correspondence should be addressed. (e-mail: martin. kuentz@fhnw.ch)
}

development, a more detailed understanding of the drug absorption process is needed. Issues of incomplete absorption, non-linear exposure and food effects should be considered early on as a function of the dose or of other formulation parameters. This refined drug absorption modeling generally requires physiologically-based pharmacokinetic models. A pioneer physiologically-based model was proposed by $\mathrm{Yu}$ et al. (18-20) It was possible to model experimental data assuming seven physiological compartments in series. Incorporation of an absorption step led to the compartmental absorption and transit model (CAT) (20). The CAT model was an important step towards physiologically-based drug absorption modeling. However, it did not include dissolution of solid particles. The simulations were therefore confined to those drugs, the dissolution of which is not rate limiting. In a further modification step to the CAT model, additional physico-chemical processes were considered. A pH-dependent solubility was introduced, as well as dissolution and precipitation steps. Drug absorption from the stomach and colon was added, as well as physiological characteristics of the absorption surface area and drug transporters. This resulting new model was called the advanced compartmental absorption transit model (ACAT) (21) and forms the basis of the commercial software GastroPlus ${ }^{\mathrm{TM}}$ (Simulations Plus, USA). A large set of differential equations can simulate the above mentioned absorption processes and a more recent version of GastroPlus $^{\mathrm{TM}}$ further includes a physiologically-based disposition model. Apart from GastroPlus ${ }^{\mathrm{TM}}$, there are additional 
integrated dynamic models available as commercial software, like the program PK-SIM $®$ (Bayer Technology Services, Germany). It is a software package for whole-body physiologically-based pharmacokinetic modeling. The underlying model is based on a homogenous tube, however, assuming a continuous intestinal transit function. It is interesting to note that the beginning of such convection-dispersion models dates back to the early 1980s (22,23). The modified convectiondispersion model (24) underlying PK-SIM $®$ was validated in rats (24), humans (25) and most recently also in monkeys (26). As an integrated model it is able to capture $\mathrm{pH}$ dependent solubility, drug dissolution, as well as the modeling of transporters and intestinal drug clearance.

Besides the ACAT and dispersion-convection models, other homogenous absorption models exist in the literature (27-29) and also a heterogeneous tube model was published $(30,31)$. However, the homogenous models are of more practical interest due to their commercial availability as software, like GastroPlus ${ }^{\mathrm{TM}}$ or PK-SIM $\AA$. The mechanistic character of the models allows testing of mechanistic assumptions. Such hypothesis driven simulations are of special interest to guide formulation development. In the preclinical phase, absorption modeling can aid defining a formulation strategy for first-in-man studies. Once initial pharmacokinetic information is available, further opportunities are given to refine model parameters. The modeling and simulation with refined parameters can further support formulation development for the clinical phases 2 and 3, or even for the market.

\section{DEFINITION OF THE CLINICAL FORMULATION STRATEGY FOR PHASE 1 STUDIES BASED ON PHYSIOLOGICAL MODELS OF DRUG ABSORPTION}

Bringing a new drug to the market is an endeavor, and biopharmaceutical issues are often revealed at a rather late stage of the development phase. Insufficient bioavailability or non-linearity and variability of drug exposure generally mean increased resource investment in formulation development. In some cases, a change of the selected formulation strategy is necessary and adhering to the original timelines is often no longer possible. Therefore, formulation changes are costly and may delay the time to market. It is evident that early anticipation of such biopharmaceutical issues can streamline the development process leading to considerable cost savings. The physiologically-based pharmacokinetic models (PBPK) are here of special interest, as it is possible to predict pharmacokinetics in man (32). The model parameters are related to the compound, as well as to the formulation. Input parameters like drug solubility, $\mathrm{pKa}$ or effective permeability can itself be calculated resulting in predictions from chemical structure alone. The comparison of such models without experiments with the results from simulations, in which measured data were used (29), showed better prediction accuracy for the latter case. Accordingly, it seems important to use measured input data for drug absorption simulations during the drug development phase. Suitable in vitro experiments can be even tailored in view of the software used. Input parameters are obtained from physico-chemical profiling of the compound, from cell culture experiments or even from animal pharmacokinetics. Due to the incertitude of these input parameters, any prediction of the human exposure before clinical Phase 1 must however be treated with caution. For this reason a procedure according to a scheme as outlined by Fig. 1 is recommended during the preclinical phase. The models used should be generally validated with respect to their physiological default parameters. A subsequent model of a new drug candidate in a pre-clinical phase should first attempt to model pharmacokinetics in an animal species. Once the in vivo results are in good agreement with the predictions, the step towards a human PBPK model can be made. The availability of such a human drug absorption model can help to define the strategy to develop a formulation for phase 1 clinical studies. This definition of a formulation strategy is particularly challenging with many poorly soluble drugs. While only limited resources should be spent at this time of the development due to the high attrition rate, the phase 1 study should not be jeopardized because of an insufficient bioavailability. Accordingly, minimal resource investment has to be balanced against a sound formulation strategy.

The development of the new drug R1315 is a good example of how modeling and simulation can guide formulation development (33). R1315 is a hydrogen sulfate, whose physico-chemical parameters are described in Table I. Due to its high lipophilicity $\left(\log \mathrm{D}_{7.4}\right.$ of 4.9$)$ and crystallinity (melting point of $241^{\circ} \mathrm{C}$ ), the drug exhibited a fairly low aqueous solubility $(<1 \mu \mathrm{g} / \mathrm{mL}$ at $\mathrm{pH}>5)$. Given the high permeability and the anticipated dose of about $160-320 \mathrm{mg} /$ day, the drug was categorized as BCS 2 (34). These preformulation results denoted the drug as a "biopharmaceutical flag". However, it was unclear if the issue was critical enough to justify the development of sophisticated formulations. Therefore a set of GastoPlus ${ }^{\mathrm{TM}}$ simulations was conducted before an extensive clinical formulation development was initiated.

Table I summarizes the simulation parameters that were used. As the estimation of the in vivo solubility is critical, R1315 was also dissolved in artificial intestinal fluids $(35,36)$ to obtain experimental values. The highest solubility of $0.2 \mathrm{mg} / \mathrm{ml}$ was obtained from simulated gastric fluid ( $\mathrm{pH}$ 1.2), whereas in fasted artificial intestinal fluid ( $\mathrm{pH} 6.5$ ) only $0.05 \mathrm{mg} / \mathrm{mL}$ could be dissolved. The obtained solubility profile was considered as a kind of "worst case", since the measurements were conducted at RT. For this reason a set of simulations was conducted with different solubility-pH profiles to scan the range of potential in vivo drug solubilization.

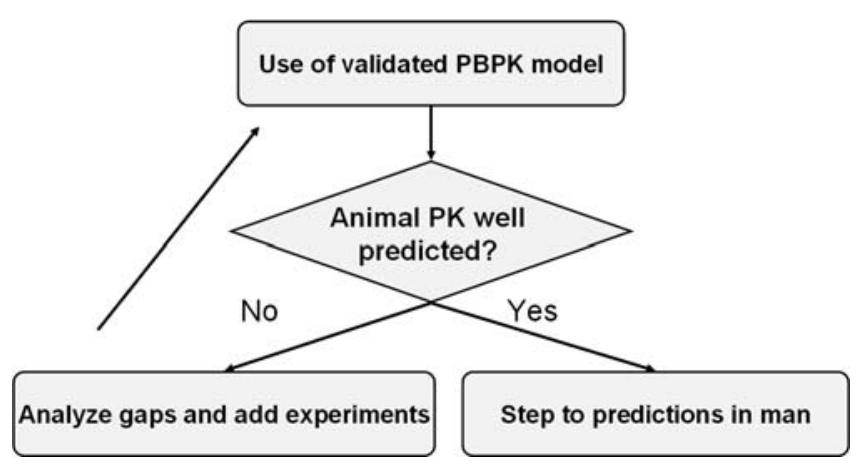

Fig. 1. Flow chart showing how to build a physiologically-based pharmacokinetic model of drug absorption in the preclinical development phase 
Table I. Input Parameters for the Drug Absorption Simulation of R1315

\begin{tabular}{lll}
\hline \multicolumn{1}{c}{ Compound Parameters } & \multicolumn{1}{c}{ Physiological Parameters } & \multicolumn{1}{c}{ PK Parameters } \\
\hline MW: $409.41 \mathrm{~g} / \mathrm{mol}$ & Human fasted condition & Body weight: $70 \mathrm{~kg}$ \\
clogP: 5.5 & Log $D$ absorption model & First pass extraction: $12.5 \%$ \\
pKa: 5.9 & Dose volume: $250 \mathrm{~mL}$ & Blood to plasma concentration ratio: 1 \\
IR capsule: $160 \mathrm{mg}$ & Stomach transit time: $0.1 \mathrm{~h}$ & Clearance estimate: $0.15 \mathrm{~L} / \mathrm{h} \mathrm{kg}$ ) \\
Lower reference solubility $(\mathrm{pH} 6.5): 0.002 \mathrm{mg} / \mathrm{mL}$ & Small intestinal transit time: $3.3 \mathrm{~h}$ & Volume of distribution: $1.9 \mathrm{~L} / \mathrm{kg}$ \\
Precipitation time: $1,800 \mathrm{~s}$ & Small intestinal radius: $1.2 \mathrm{~cm}$ & - \\
Particle density: $1.2 \mathrm{~g} / \mathrm{mL}$ & Small intestinal length: $300 \mathrm{~cm}$ & - \\
Effective permeability: $4.4 \times 10^{-4} \mathrm{~cm} \mathrm{~s}^{-1}$ & Colon transit time $18 \mathrm{~h}$ & - \\
Mean particle size: $5 \mu \mathrm{m}$ & Colon volume: $1,200 \mathrm{~mL}$ & - \\
\hline
\end{tabular}

The predicted human bioavailability was simulated by means of a parameter sensitivity analysis (PSA). Interestingly, the simulations showed (Fig. 2) that this parameter was essentially unchanged in the relevant solubility and particle size ranges. Another PSA at a higher dose of $320 \mathrm{mg}$ returned similar simulation data. These results confirmed the BCS class 2 status for R1315, and that within the relevant solubility and particle size ranges, no dramatic exposure changes were to be anticipated.

A mechanistic interpretation can be obtained from the simulation plot (Fig. 3), in which the dissolved drug is shown as a function of time. As expected, a considerable fraction of the dose is first solubilized followed by precipitation in the intestine. However, the transit time for the given doses seemed long enough to allow for complete absorption. In light of these results additional simulations were conducted by varying the drug's precipitation time from 180 to $18,000 \mathrm{~s}$, which again did not greatly affect the predicted exposure. Thus, the model predicted that drug absorption was rather insensitive within the relevant dose range and formulation parameters.

The simulation results provided no rational for a resource-intensive development of specialized formulations

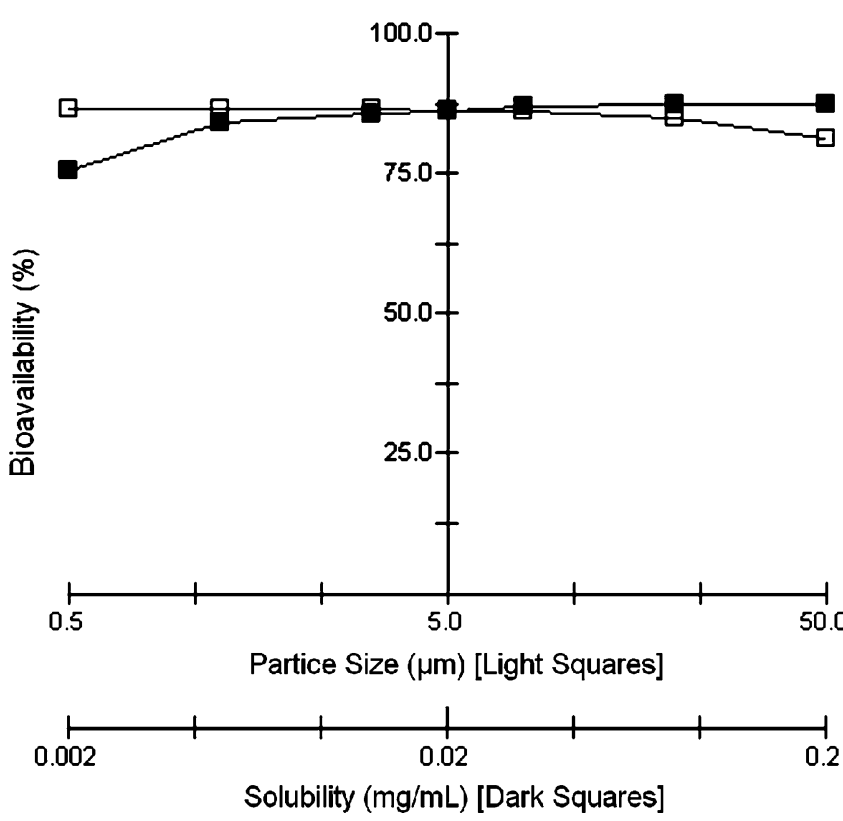

Fig. 2. Parameter sensitivity analysis of the oral bioavailability following a dose of $160 \mathrm{mg} \mathrm{R} 1315$ like solid dispersions or lipid delivery systems. The hypothesis was made that a standard solid dosage form could be developed for first studies in man. Prior to this phase 1 formulation development, a simple drug powder blend was compared with a micellar drug solution in dog studies. The results showed no significant difference between these formulations in the relevant dose range (33). This supported the choice of a standard formulation for phase 1 and demonstrated how strategic formulations decisions can be made based on PBPK simulations.

A different case was presented by the drug LAB687 from Novartis (New Jersey, USA) (37). The neutral compound with a MW of $468.5 \mathrm{~g} / \mathrm{mol}$ and a $\log P$ of 4.7 had an aqueous solubility of about $0.17 \mu \mathrm{g} / \mathrm{mL}$. This value was
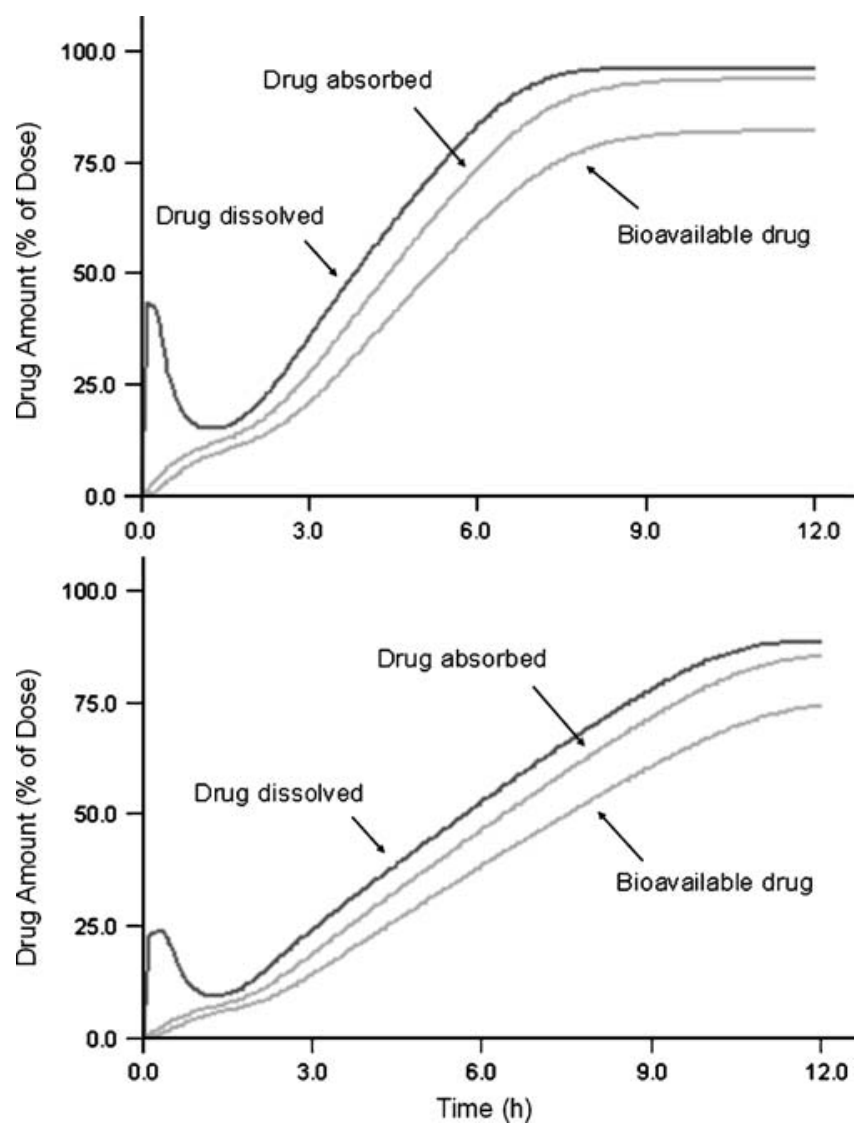

Fig. 3. Modelling of the different drug states upon intestinal absorption as a function of time for $160 \mathrm{mg}$ (upper figure) and 320 mg R1315 (lower figure) 
expected to be higher in intestinal fluids and a value of $1 \mu \mathrm{g}$ / $\mathrm{mL}$ was obtained from tests in a bile salt solution. Similar to the previous example, GastoPlus ${ }^{\mathrm{TM}}$ simulations were run for different parameter settings in a dose range of 5-500 $\mathrm{mg}$. However, the PSA showed some ranges of high exposure sensitivity for LAB687 based on the solubility of the drug, as well as a dependence on the particle size. As the inspected ranges were of technological relevance the simulations raised concerns for the upcoming formulation development. The in silico results were also in this case complemented by tailormade pharmacokinetic studies in the dog. A standard powder blend of the drug (micronized) in capsules was compared with two other formulations. The rationale for the latter formulations was to increase the in vivo solubilization of the drug. Accordingly, a co-solvent/surfactant solution of Cremophor was tested together with a solid dispersion of the drug in a mixture of 3:1 PEG3350 to polysorbate 80 . Table II exhibits the results of this dog PK study. Both biopharmaceutically enhanced formulations exhibited about 10x higher oral bioavailability compared to the dry powder blend. Accordingly, the in vivo study in the dog model agreed with the in silico prediction with respect to the biopharmaceutical hurdle of LAB687. A strong rationale was given to invest adequate resources in the formulation development.

The two case studies of the new drugs R1315 and LAB687 both demonstrate how modeling and simulation of drug absorption can provide a rational basis for the formulation development strategy. The physiologically-based models used in both cases measured compound properties to estimate the pharmacokinetic behavior in humans based on existing animal data. These adapted physiologically-based models have a higher quality of input parameters as compared to generic physiologically-based models that are used in the clinical candidate selection phase. During the course of the development, the level of information on the new drug increases and this can be reflected by a higher model predictability. However, before the drug is tested in humans there is always a given level of incertitude. Parrott et al. (2006) (32) reviewed the predicted versus the observed AUC of 19 Roche compounds. Interestingly, $76 \%$ of the predictions using the physiologically-based modeling approach was within a two-fold range of the observed results. This accuracy seems good enough to anticipate the human exposure and to guide decision-making during the development of a phase 1 formulation.

\section{MODELING OF FACTOR INTERACTIONS TO SUPPORT FORMULATION DEVELOPMENT}

A major step in the development of a drug as well as in the evolution of an absorption model is the first testing in man. The availability of human exposure data opens new doors for the modeling capabilities, leading for example to the model's next level of complexity. Tubic et al. (38) proposed an Advanced Compartmental and Transit model for the absorption of the drug Talinolol. P-glycoprotein (Pgp)-mediated efflux for this drug was assumed, which needed an estimation of the transport parameters $K_{\mathrm{m}}$ and $V_{\max }$. The adapted physiologically-based model captured well the nonlinearity of the exposure with increasing doses between 25 and $400 \mathrm{mg}$. The authors also performed a set of simulations in a virtual clinical trial. Accordingly, the drug absorption simulation estimated not only the exposure for a given dose, but provided further a variability forecast. This information is especially valuable for the later stages of the formulation development. The target specifications of the formulation could be set on a more rational basis, which could help, for example, in attempts to increase the bioavailability of a formulation. The simulations could compare a targeted formulation effect with the anticipated inter-subject variability resulting in a clear view on what can be achieved by means of the formulation.

A reality-check of a human absorption model occurs when the drug product enters its first clinical trials. Having human data of a drug candidate can not only be used to assess the predictability of the model, but may also feed-back to the process of how to find a model in the preclinical phase. Thus, in vitro tests can be improved with respect to estimating simulation parameters. Recently physiologically-based modeling was used to establish an in vitro/in vivo correlation (IVIVC) for the BCS class 2 drug glyburide (39). Such computer models could potentially be used as surrogate for bioequivalence studies. The risks of a study failure could be better anticipated in advance, which is of great importance for the later clinical phases or for generic formulation development.

Once clinical data is available, it is possible to revisit the in vitro results used for the absorption modeling. Experimental testing conditions may be improved in the light of human exposure data. Thus, a recent article (40) questioned the most widely used composition of simulated gastric fluid (41). The critique originated from physico-chemical concerns and was supported by an absorption modeling using GastroPlus ${ }^{\mathrm{TM}}$. A new composition of simulated gastric fluid was proposed (40) and accordingly, the modeling can be used to further improve the recipe of simulated intestinal fluids.

The presented examples demonstrate that physiologically-based modeling offers a variety of tools for the formulator in the later stages of the formulation development. It seems that the full potential of these approaches has not yet been exploited by the industry. Thus, the last case study of this article presents another application of drug absorption modeling at later stages of the formulation development. Previous examples showed that a single simulation is of less interest compared to a set of simulations using PSA. It is the varying exposure depending on changes of technical parameters that is critical for the formulation development. The

Table II. Mean Pharmacokinetic Parameters and Their Relative Bioavailability in the Dog Studies for the Three Formulation Approaches

\begin{tabular}{|c|c|c|c|c|}
\hline $50 \mathrm{mg}$ LAB687 of formulation & $T_{\max }(\mathrm{h})$ & $C_{\max }(\mu \mathrm{g} / \mathrm{mL})$ & $\mathrm{AUC}_{0-48 \mathrm{~h}}(\mu \mathrm{g} \mathrm{h} / \mathrm{mL})$ & $F_{\text {rel }}(\%)$ \\
\hline Surfactant solution & $1-2$ & 1.16 & 6.96 & 100 \\
\hline Solid dispersion & $1-2$ & 0.803 & 6.90 & 99.1 \\
\hline Dry blend & 1 & 0.128 & 0.681 & 9.8 \\
\hline
\end{tabular}




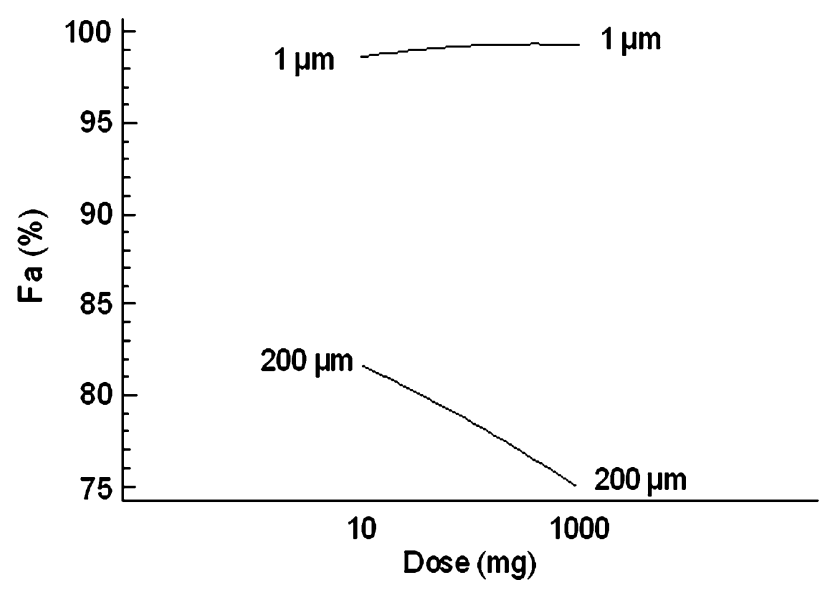

$(X 0.001)$

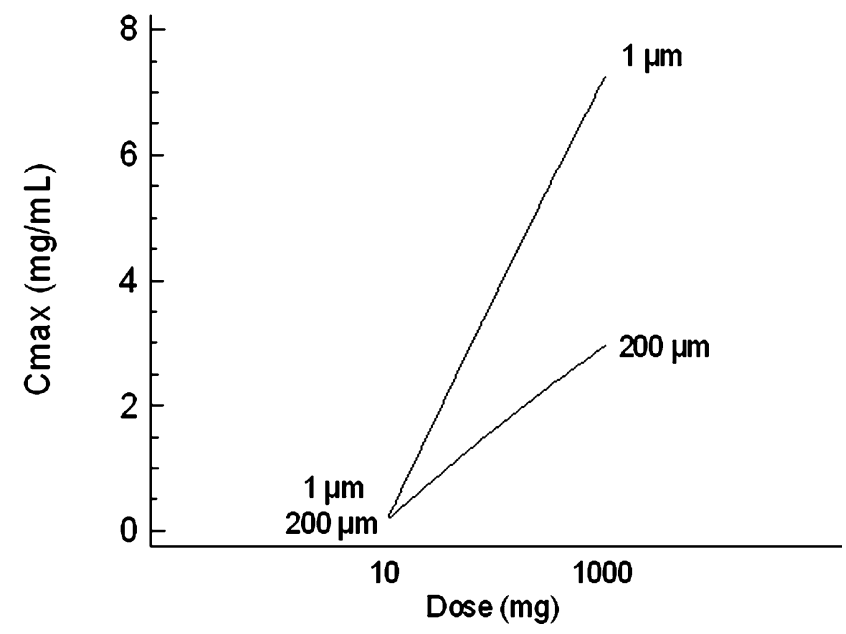

Fig. 4. Interaction plot of dose and particle size of mefenamic acid with respect to the fraction absorbed and maximal plasma concentration, respectively

standard PSA starts from a given average value and conducts simulations by varying one parameter at a time. The routine can be repeated with other parameters and the results are typically presented in a plot like Fig. 2. As good as the procedure is to study factor influences, it is not adequate to reveal factor interactions. Such an interaction exists when a given factor depends on the level of another. To study factor interactions, the computer experiments are conducted according to a special design. Such statistical designs are often used in experimental pharmaceutics to study factors, interactions, and response surfaces $(42,43)$. The conduct of computer experiments according to a statistical design goes beyond the single factor PSA so that an entire drug absorption design space (DADS) can be evaluated. The response surface may be mapped according to statistical model equations and in the future the physiologically-based software may calculate each point of the response surface by an automated routine. The following example shows the principle of DADS using mefenamic acid as a model substance.

The program PK-SIM $®$ employing a modified convection-dispersion model (24-26) was used for these simulations. As compound properties for mefenamic acid, we set a MW of $241.3 \mathrm{~g} / \mathrm{mol}, \mathrm{a} \log P$ of 3.52 , a $\mathrm{pKa}$ (apparent) of 6.4 and a solubility of $46 \mu \mathrm{g} / \mathrm{mL}$ ( $\mathrm{pH} 5.5$ ). The program calculated an intestinal effective permeability of $2.68 \times 10^{-5} \mathrm{~cm} / \mathrm{s}$, using as default physiological parameters of the program (version 3.0) the fasted human state. A fraction unbound of $1 \%$ in combination with a clearance value of $0.26 \mathrm{l} /(\mathrm{h} \mathrm{kg})$ (44) was set for all simulations of mefenamic acid.

The initial absorption simulation was performed at an oral dose of $500 \mathrm{mg}$ with an average drug particle size of $100 \mu \mathrm{m}$. This average particle size of a monodisperse distribution was a first approximation of the real particle size distribution, which was essentially polydisperse (45). Thus, a simple model of the unmilled drug was obtained and the predicted plasma profiles were compared with the published data from a clinical study (45). The plasma profiles turned out to be in good agreement with the theoretical predictions. Based on this successful model verification we wanted to study the effects of dose and particle size, as well as their interaction in relation to the oral drug exposure.

A four level statistical design $\left(4^{2}\right)$ was conducted with rather broad factor ranges. The doses were set at 10, 340, 670, and $1,000 \mathrm{mg}$ and the particle size ranged from 1, 67.33, 133.67 to $200 \mu \mathrm{m}$. The simulation results were analysed with respect to the fraction absorbed, $F_{\mathrm{a}}$ and $C_{\max }$ using a statistical software package (Statgraphics Centurion XV). The factors dose and particle size were found to be highly significant for $F_{\mathrm{a}}$ as well as for $C_{\max }(p<0.0001)$. Interestingly, the interaction of the two factors was also highly significant $(p<0.0001)$ for both responses. Fig. 4 highlights this finding by means of an interaction plot. The lower particle sizes result in practically complete absorption for the extreme doses of 10 as well as 1,000 mg. However, at a particle size of $200 \mu \mathrm{m}$, the
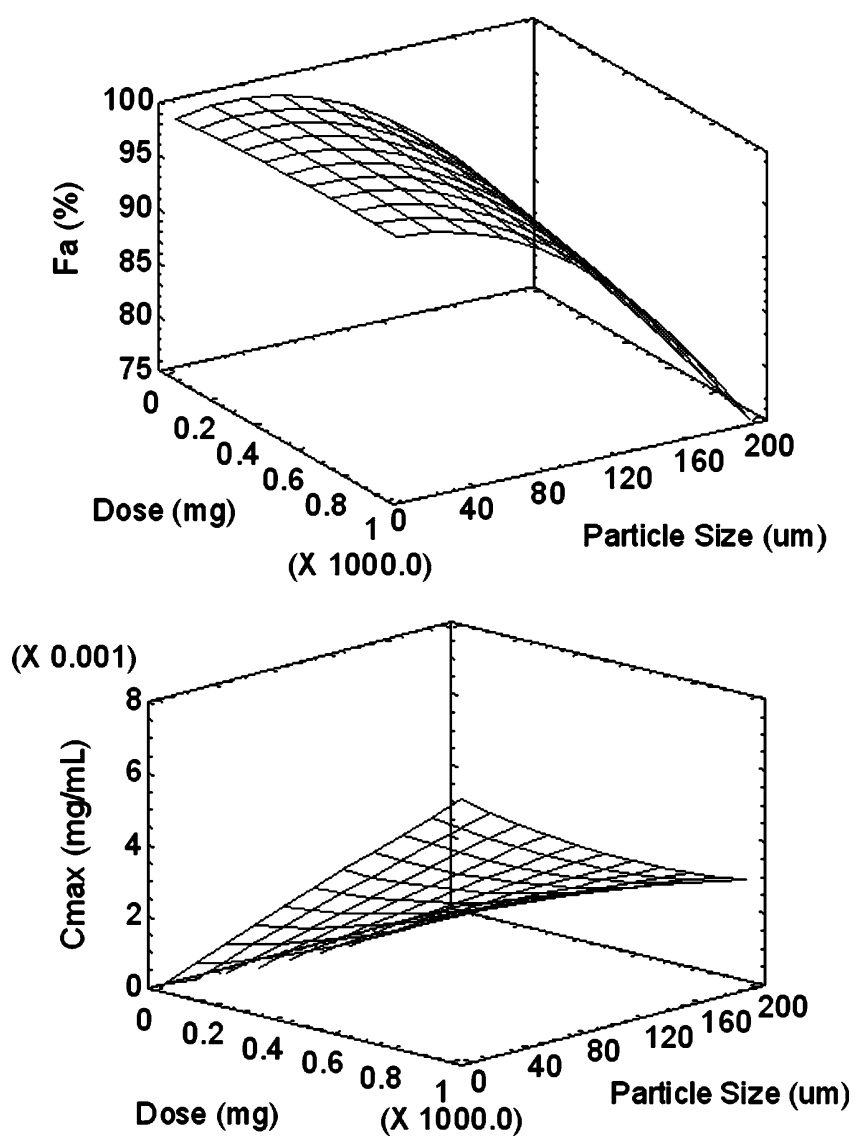

Fig. 5. Response surface plot of the modelled dose fraction absorbed and maximal plasma concentration using mefenamic acid as model 
fraction absorbed was incomplete and there was a substantial difference between the two doses. Accordingly, the dose effect on drug absorption only comes into existence when coarser particles lead to limited dissolution of the drug. Fig. 4 displays also the interaction with respect to the maximal plasma concentration. The lower dose, being less critical for complete dissolution of the drug, showed no particle size effect. However, a pronounced particle size effect was observed when the dose increased to the higher level of $1,000 \mathrm{mg}$. The higher dose level was critical from an exposure perspective, which was also reflected by the response surface in Fig. $5\left(R^{2}=0.998\right.$ for $F_{\mathrm{a}}$ and $R^{2}=0.996$ for $\left.C_{\max }\right)$. A plateau of complete absorption was observed within a small particle size range. However, at a higher particle size, a steep drop of the absorbed dose fraction was predicted.

The modeling results provide a rational answer to a typical question in drug development. Should the drug be milled, or should the unmilled drug be used to save costs? The model demonstrated that an answer from a biopharmaceutical perspective must be given in relation to the dose. For higher doses of mefenamic acid the model indicated that milling is required. It seems reasonable to stay clearly in the plateau range of complete absorption, since in a steeper part of the response surface erratic absorption as well as high inter-subject variability must be expected. The plateau denotes therefore a formulation robustness with respect to dosage and average particle size. This robust range can also be observed by inspecting the maximal plasma concentrations, $C_{\max }$. Strict linearity for the dose was only predicted within this range of complete drug absorption (Fig. 5).

In line with our findings based on this model drug, the authors Hummel and Buchmann (45) found that for a generic formulation, the milling of mefenamic acid significantly raised drug absorption and bioavailability, respectively. Batches of unmilled drug were shown to exhibit a rather course particle size distribution. The slower dissolution from the coarser drug was associated with erratic drug absorption and incomplete bioavailability.

It was argued at the beginning of this decade that a bioavailability study was indispensable for generic formulations of mefenamic acid. Relying on dissolution data alone was considered as not being sufficient for this model drug. However, today the physiologically-based drug absorption modeling can integrate the critical drug factors for example the dose or particle size to construct a DADS. Critical ranges of the drug and of formulation factors may be distinguished from other parameter ranges, where complete absorption is anticipated. In the latter part of the DADS, there is no relevant bioavailability variance expected when comparing different formulations. Thus, a well founded DADS may in the future lead to reduced or even omitted clinical bioavailability testing.

\section{CONCLUSIONS}

Physiologically-based models go far beyond the sole purpose of prediction of drug absorption. Their mechanistic character is of particular advantage once more information is available on a development compound. The quality of input parameters can at that point be improved and mechanistic hypotheses be checked by specially designed experiments.
This procedure can guide the preclinical formulation development. The biopharmaceutical hurdles of the drug can be anticipated early on and a rational may be given for a more complex formulation development. In this context the parameter sensitivity analysis is particularly useful. It can predict exposure based on changes in the compound or formulation parameters. This concept has the potential to be further extended to a systematic screening of a drug absorption design space. Factor interactions become apparent as well as ranges, wherein a formulation appears to be robust from a biopharmaceutical perspective. Accordingly, physiologically-based modeling offers a variety of tools for a pharmacist and an excellent opportunity is given for a more focused and thus cost-effective formulation development.

\section{REFERENCES}

1. P. Cuatrecasas. Drug discovery in jeopardy. J. Clin. Invest. $\mathbf{1 1 6}$ (11):2837-2842 (2006).

2. I. Kola, and J. Landis. Can the pharmaceutical industry reduce attrition rates? Nature Reviews Drug Discov. 3(8):711-715 (2004).

3. J. Wang, and L. Urban. The impact of early ADME profiling on drug discovery and development strategy. Drug Discov. 4:73-86 (2004).

4. H. Van de Waterbeemd, and E. Gifford. ADMET in silico modeling: Towards prediction paradise? Nat. Rev. 2(3):192-204 (2003).

5. F. Lombardo, E. Gifford, and M. Y. Shalaeva. In silico ADME prediction: Data, models, facts, myths. Minirev Med. Chem. 3 (8):861-875 (2003).

6. C. A. Lipinski, F. Lombardo B. W. Dominy et al. Experimental and computational approaches to Estimate solubiliy and permeability in drug discovery and development settings. Adv. Drug. Deliv. Rev. 23(1-3):3-25 (1997).

7. C. W. Andrews, L. Bennett, and L. X. Yu. Predicting human oral bioavailability of a compound: development of a novel quantitative structure-bioavailability relationship. Pharm. Res. 17 (6):639-44 (2000).

8. F. Yoshida, and J. G. Topliss. QSAR model for drug human oral bioavailability. J. Med. Chem. 43(13):2575-2585 (2000).

9. D. F. Veber, S. R. Johnson, H. Y. Cheng, B. R. Smith, K. W. Ward, and K. D. Kopple. Molecular properties that influence the oral bioavailability of drug candidates. J. Med. Chem. 45:26152623 (2002).

10. M. A. C. Perez, M. B. Sanz, L. R. Torres, R. G. Avalos, and M. P. Gonzalez. Diaz HGA topological sub-structural approach for predicting human intestinal absorption of drugs. Eur. J. Med. Chem. 39(11):905-916 (2004).

11. E. Deconinck, Q. S. Xu, R. Put, D. Coomans, D. L. Massart, and Y. Vander Heyden. Prediction of gastro-intestinal absorption using multivariate adaptive regression splines. J. Pharm. Biomed Anal. 39(5):1021-1030 (2005).

12. E. Deconinck, H. Ates, N. Callebaut, E. Van Gyseghem, and Y. Vander Heyden. Evaluation of chromatographic descriptors for the prediction of gastro-intestinal absorption of drugs. $J$. Chromatogr., A. 1138(1-2):190-202 (2007).

13. T. Hou, J. Wang, W. Zhang, and X. Xu. ADME evaluation in drug discovery. 7. Prediction of oral absorption by correlation and classification. J. Chem. Inf. Model. 47(1):208-218 (2007).

14. T. Hou, J. Wang, and Y. Li. ADME evaluation in drug discovery. 8. The prediction of human intestinal absorption by a support vector machine. J. Chem. Inf. Model. 47(6):2408-2415 (2007).

15. T. Hou, J. Wang, W. Zhang, and X. Xu. ADME evaluation in drug discovery. Can oral bioavailability in humans be effectively predicted by simple molecular property-based rules? J. Chem. Inf. Model. 47(2):460-463 (2007).

16. J. Dressman, G. Amidon, and D. Fleisher. Absorption potential: Estimation of the fraction absorbed for orally administered compounds. J. Pharm. Sci. 74(5):588-589 (1985). 
17. K. Johnson, and A. Swindell. Guidance in the setting of drug particle size specifications to minimize variability in absorption. Pharm. Res. 13(12):1795-1798 (1996).

18. L. Yu, J. Crison, and G. Amidon. Compartmental transit and dispersion model analysis of small intestinal transit flow in humans. Int. J. Pharm. 171(2):157-163 (1996).

19. L. Yu, and G. Amidon. Characterisation of small intestinal transit time distribution in humans. Int. J. Pharm. 171(2):157-163 (1998).

20. L. Yu, and G. Amidon. Saturable small intestinal drug absorption in humans: Modeling and interpretation of cefatrizine data. Eur. J. Pharm. Biopharm. 45(2):199-203 (1998).

21. B. Agoram, W. Woltosz, and M. Bolger. Predicting the impact of physiological and biochemical processes on oral drug bioavailability. Adv. Drug Deliv. Rev. 50(S1):S41-67 (2001).

22. P. Ni, N. Ho, J. Fox, H. Leuenberger, and W. Higuchi. Theoretical model studies of intestinal drug absorption V. Non-steady-state fluid flow and absorption. Int. J. Pharm. 5(1):33-47 (1980).

23. N. Ho, J. Park, P. Ni, and W. Higuchi. Advancing quantitative and mechanistic approaches in interfacing gastrointestinal drug absorption studies in animals and humans. In W. Crouthamel and A. Sarapu (eds.), Animal Models for Oral Drug Delivery. In Situ and In Vivo Approaches, American Pharmaceutical Association, Washington, 1983, pp. 27-106.

24. S. Willmann, W. Schmitt, J. Keldenich, and J. Dressman. A physiological model for simulating gastrointestinal flow and drug absorption in rats. Pharm. Res. 20(11):1766-1771 (2003).

25. S. Willmann, W. Schmitt, J. Keldenich, J. Lippert, and J. Dressman. A physiological model for the estimation of the fraction dose absorbed in humans. J. Med. Chem. 47(16):40224031 (2004).

26. S. Willmann, A. N. Ediginton, and J. Dressman. Development and validation of a physiology-based model for the prediction of oral absorption in monkeys. Pharm. Res. 24(7):1275-1282 (2007).

27. G. M. Grass. Simulation models to predict oral drug absorption from in vitro data. Adv. Drug Deliv. Rev. 23:199-219 (1997).

28. D. A. Norris, G. D. Leesman, P. J. Sinko, and G. M. Grass. Development of predictive pharmacokinetic simulation models for drug discovery. J. Controlled Release. 65:55-62 (2000).

29. N. Parrott, and Th. Lavé. Prediction of intestinal absorption: comparative assessment of GastroPlusä and IDEAä. Euro. J. Pharm. Sci. 17:51-61 (2002).

30. A. Kalampokis, P. Argyrakis, and P. Macheras. Heterogenous tube model for the study of small intestinal transit flow. Pharm. Res. 16(1):87-91 (1999).

31. A. Kalampokis, P. Argyrakis, and P. Macheras. A heterogenous tube model of intestinal drug absorption based on probabilistic concepts. Pharm. Res. 16(11):1764-1769 (1999).

32. N. Parrott, H. Jones, N. Paquereau, and T. Lavé. Application of full physiological models for pharmaceutical drug candidate selection and extrapolation of pharmacokinetics to man. Basic \& Clin. Pharmacol. Toxicol. 96:193-199 (2005).

33. M. Kuentz, S. Nick, N. Parrott, and D. Roethlisberger. A strategy for preclinical formulation development using GastroPlusä as pharmacokinetic simulation tool and a statistical screening design applied to a dog study. Eur. J. Pharm. Sci. 27(1):91-99 (2006).

34. G. Amidon, H. Lennernas, V. Shah, and J. Crison. A theoretical basis for a biopharmaceutical drug classification: The correlation of in vitro drug product dissolution and in vivo bioavailability. Pharm. Res. 12(3):413-420 (1995).

35. E. Galia, E. Nicolaides, D. Hörter, R. Löbenberg, Ch. Reppas, and J. Dressman. Evaluation of various dissolution media for predicting in vivo performance of class I and II drugs. Pharm. Res. 15(5):698-705 (1998).

36. M. Vertzoni, N. Fotaki, E. Kostewicz, E. Stippler, Ch. Leuner, E. Nicolaides, J. Dressman, and Ch. Reppas. Dissolution media simulation the intralumenal composition of the small intestinal intestine: physiological issues and practical aspects. J. Pharm. Pharmacol. 56:453-562 (2004).

37. R. M. Dannenfelser, H. He, Y. Joshi, S. Bateman, and A. Serajuddin. Development of clinical dosage forms for a poorly water soluble drug I: application of polyethylene glycol-polysorbate 80 solid dispersion carrier system. J. of Pharm. Sci. 93 (5):1165-1175 (2004)

38. M. Tubic, D. Wagner, H. Spahn-Langguth, M. Bolger, and P. Langguth. In silico Modeling of non-linear drug absorption for the P-gp substrate talinolol and of consequences for the resulting pharmacodynamic effect. Pharm. Res. 23(8):1712-1720 (2006).

39. H. Wei, and R. Löbenberg. Biorelevant dissolution media as a predictive tool for glyburide a class II drug. Euro. J. Pharm. Sci. 29:45-52 (2006).

40. A. Aburub, D. S. Risley, and D. Mishra. A critical evaluation of fasted state simulating gastric fluid (FaSSGF) that contains sodium lauryl sulfate and proposal of a modified recipe. Int. $J$. Pharm. 347:16-22 (2008).

41. J. Dressman, G. Amidon, C. Reppas, and V. Shah. Dissolution testing as a prognostic tool for oral drug absorption: immediate release dosage forms. Pharm. Res. 15:11-22 (1998).

42. G. A. Lewis, D. Mathieu, and R. Phan-Tan-Luu. Pharmaceutical Experimental Design. Marcel Dekker, New York, 1999, pp 27-184.

43. M. Kuentz, D. Röthlisberger, and W. Richter. Design of experiment (DoE) methods maximize information from a minimal number of animals in special cases of preclinical bioavailability testing. Pharm. Dev. Technol. 8(4):453-458 (2003).

44. C. Dollery. Mefenamic Acid. In Therapeutic Drugs. 2nd edn. Churchill Livingston, Edinburg, London, 1998, pp M21-M24.

45. D. Hummel, and St. Buchmann. Influence of the particle size of mefenamic acid on dissolution and bioavailability of tablets (article in German). Pharm. Ind. 62(6):452-456 (2000). 